\title{
3D NUMERICAL MODELING OF THE CRACK-PERMEABILITY INTERACTION IN FRACTURED CONCRETE
}

\author{
M. MATALLAH* , C. LA BORDERIE ${ }^{\dagger}$ \\ *RiSAM, University of Tlemcen \\ Algeria, \\ e-mail: matallah@mail.univ-tlemcen.dz \\ ${ }^{\dagger}$ SIAME, University of Pau, \\ France \\ e-mail: christian.laborderie@ univ-pau.fr
}

Key words: Concrete, Permeability, Cracks, Poromechanics

\begin{abstract}
In the present work, a coupled mechanical-hydraulic-based approach is proposed for numerical modeling of the interdependent mechanical and hydraulic behavior of fractured concrete. The proposed model is developed within the framework of the non linear poromechanics. A damage based model is used to describe the mechanical behavior of the solid phase (the solid skeleton). Crack estimation is, therefore, performed using a post-processing method based on the fracture energy regularization. The hydraulic behavior is governed by the Darcy's law for the uncracked material. After cracking, the flow through fracture is driven by the cubic law and an anisotropic description of the material permeability is, therefore, assumed. The validation is performed on a splitting test where a real-time crack-permeability interaction is assessed. The capability of the proposed model to accurately reproduce the experimental results is proven.
\end{abstract}

\section{Introduction}

Flow and transport in fractured porous media is an important field of great interest in industrial applications. In the context of nuclear industry, concrete is used both for the construction of nuclear power plant and the waste packages. The fracture process provides preferential pathways for fluid migration through concrete increasing its permeability. Therefore, understanding the fluid flow mechanism through porous and cracked concrete is important for assessing the leak-tightness. Analytic and numerical approaches should be considered to assess the coupling between the evolution of the cracking process and the permeability. The coupling between damage, cracking and permeability has been already investigated. Experimen- tal tests performed on concrete specimens under compression loading (axial compression [1] or triaxial [12]) show that distinguished regimes are observed regarding the permeability evolution. The same observations have been established using direct tensile tests [7] or splitting test [21]. The permeability-increases depends on the evolution of the crack openings. Within the framework of damage mechanics, various authors have proposed to relate the permeability evolution to the damage variable [4] or using two distinguished regimes, a diffused one relating the permeability to the damage variable and a localized one driven by the crack opening [5]. Experimental results issued from direct tensile tests show that the permeability is directly related to the crack aperture. Therefore, the crack opening estimation is the key of the 
permeability crack interaction modelling. Also, some phenomena (Crack closure effect, Cyclic loading ..) could not be taken into account using the approaches based on a damage-permeability interaction.

The objective of this paper is to study the crack-permeability interaction. The proposed approach is firstly described. In order to give a discrete representation of the fracture properties in space (opening, propagation, ..), an energetic based approach proposed by the authors [11] is used. The cracking process is furthermore represented through a second order tensor (The Unitary Crack Opening Tensor) [10] which permits an anisotropic description of the fractured medium. The description of the flow and the transport process in porous uncracked concrete is described by the wellknown Darcy equation [3]. When cracks occur, the physical-mathematical description of laminar flow through fractures is described by the cubic-law [17] developed using the ParallelPlate Concept [20] [22]. The proposed approach is developed within the framework of the non linear poromechanics. The validations are performed on a Brazilian splitting test. Comparisons with experimental data are illustrated.

\section{Fundamental Basis}

The proposed approach is developed within the framework of the Biot theory of poromechanics. The porous medium has two phases : the solid skeleton and the moving pore fluid. When dealing with the modelling of flow transport in fractured media, investigations are associated with the scale considered for the description. In general, these scales are microscopic (pore scale) or macroscopic (field scale). The coupling approach proposed here is developed considering a continuum scale and using macroscopic quantities 1 . The governing equation are written in terms of macroscopic parameters and properties. The fractured medium with discontinuities (cracks) is considered a continuum fractured medium. The following dynamic and kinematic parameters are used : $\sigma_{i j}$ the total stress tensor and $\varepsilon_{i j}$ the strain tensor of the porous frame, the pore pressure $p$ which is a scalar and its kinematic dual variable $\xi$ which represents the variation of fluid content.

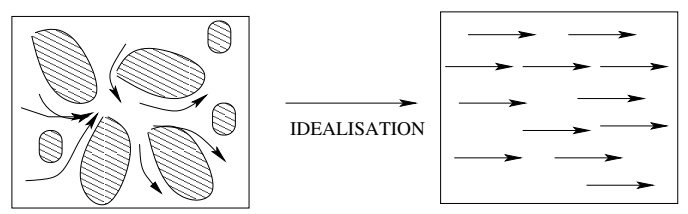

Figure 1: A continuum medium with two continuum phases

\section{Governing equations}

\subsection{Equilibrium equations}

The equilibrium equation reads

$$
\operatorname{div} \sigma+F=0
$$

and the continuity equation of the fluid phase reads

$$
\frac{\partial \xi}{\partial t}+\operatorname{div} q=\gamma
$$

the term $q$ is the rate of fluid volume crossing a unit area of porous solid and $\gamma$ is the source terms relating to the rate of injected fluid volume per unit volume of the porous solid.

\subsection{Constitutive Laws}

The stress-strain relations for the solid phase is given by

$$
\sigma_{i j}=C_{i j k l} \varepsilon_{k l}-B_{i j} p
$$

where the first part $C_{i j k l} \varepsilon_{k l}$ represents the effective stress tensor, ie, the stress tensor in the solid phase (The effective stress tensor concept leading to the Terzaghi effective stress for $B_{i j}=\delta_{i j}$ ).

$C_{i j k l}$ is a fourth order tensor. Using a damage model, $C_{i j k l}$ is related to the damage variable. $B_{i j}$ is a the Biot coefficients tensor ( a second-order tensor).

Equation 3 expresses the evolution of the total-stress tensor components. The evolution for the other dynamic variable (the pore pressure) is given by :

$$
p=M\left(\xi-B_{i j} \varepsilon_{i j}\right)
$$


where $\mathrm{M}$ is the Biot Modulus.

\section{Solid and fluid behaviors}

The solid phase bahavior is described by a damage model. A non linear damage behavior is considered and the stress-strain governing equation for the solid skeleton is given by [6]

$$
\sigma_{s}=C_{i j k l}^{0}(1-d) \varepsilon_{k l}
$$

$C_{i j k l}^{0}$ is the undamaged fourth order tensor. A scalar damage variable is considered. The damage evolution law integrated is given by :

$$
d=1-\frac{\varepsilon_{d 0}}{\varepsilon} \exp \left(B\left(\varepsilon_{d 0}-\varepsilon\right)\right)
$$

where $B$ is a parameter which controls the slope of the softening curve defined by the exponential expression and $\varepsilon_{d 0}$ the strain threshold (ie the threshold triggering softening).

Under tensile loading (Mode I), the softening behavior is governed by the fracture energy parameters. The fracture energy is, therefore, given by

$$
G_{f}=h \frac{E * \varepsilon_{d 0}}{B}
$$

where $E$ the Young's modulus with $f_{t}=E * \varepsilon_{d 0}$ and $h$ is the finite element size related to the localized band-width. This leads to a meshindependent energy release upon crack propagation in Mode I under tensile stress (See [11] [9]).

For the second phase, a macroscopic description of the fluid transport in the pore could be achieved using the Darcy Law :

$$
q=-\frac{k}{\mu} \nabla P
$$

where the body force per unit volume of fluid are neglected, $k$ is the intrinsic permeability function of the porosity of the solid porous (which does not depend on the fluid properties), $\mu$ is the fluid dynamic viscosity. The fraction $K=\frac{k}{\mu}$ represents the material conductivity. The Darcy Law could also be written as

$$
Q=-k \frac{A}{\mu} \nabla P
$$

The above equations are valid under the restriction that the permeability is a number. Under isotropic considerations, the hydraulic conductivity (or the intrinsic permeability) is described by a scalar variable. Uncracked concrete is often described by a "scalar" permeability. However, under loading, cracks occur allowing more fluid penetration through preferential paths. Therefore, anisotropic hydraulic behavior should be considered. The flow through cracks is described in the next section.

\section{Crack-Permeability interactions}

\subsection{Crack openings computation}

A practical method to estimate crack opening from a finite element computation based on damage and/or plastic model has been developed by the authors in [11]. This method is proposed in the Finite Element code Cast3M (Procedure OUVFISS). The method is based on the fracture energy regularization. The crack openings evaluation concerns the concrete solid phase. From a finite element computation based a non linear damage/plasticity model, we could compute the stress tensor $\sigma_{s}$ (Equation 5). The elastic stress is computed using the total strain Eq. (10)

$$
\sigma_{i j}^{e}=C_{i j k l}^{0} \varepsilon_{k l}
$$

The total strain in the solid skeleton $\varepsilon$ is decomposed into two parts : an elastic part $\varepsilon^{e}$ and a cracking part represented by the Unitary Crack Opening strain tensor $\varepsilon^{u c o}[10]$.

$$
\varepsilon_{i j}=\varepsilon_{i j}^{e}+\varepsilon_{i j}^{u c o}
$$

Multiplying (11) by the undamaged elastic stiffness tensor $C_{i j k l}^{0}$, we obtain :

$\sigma_{i j}^{e}=C_{i j k l}^{0} \varepsilon_{k l}=C_{i j k l}^{0} \varepsilon_{k l}^{e}+C_{i j k l}^{0} \varepsilon_{k l}^{u c o}=\sigma_{i j}^{s}+\sigma_{i j}^{i n}$

The tensor of the crack openings strain is the dual variable of the inelastic stress tensor and is given by :

$$
\varepsilon_{i j}^{u c o}=\left(C_{i j k l}^{0}\right)^{-1} \sigma_{i j}^{i n}
$$

The inelastic stress tensor is, therefore, given by :

$$
\sigma_{i j}^{i n}=\sigma_{i j}^{e}-\sigma_{i j}^{s}
$$


Eq. (13) gives the Unitary Crack Opening strain tensor. The value of the normal crack opening displacement is given by :

$$
\delta_{n}=n_{i} \delta_{i j} n_{j}=n_{i} h \varepsilon_{i j}^{u c o} n_{j}
$$

Where $n$ is the unit vector normal to the crack and $h$ the length over which the crack is supposed smeared. The method has been validated under different arbitrary loadings and complex boundary conditions [11], [18], [13].

\subsection{Evolution of the permeability tensor}

Flow through non-fractured porous media occurs at the micro-scale through interstitial pores. This process is well described by the Darcy law. As it has been outlined in the introduction, when cracks occur, the physical-mathematical description of laminar flow through fractures is described by the cubiclaw developed using the Parallel-Plate Concept [20] [22](Figure 2)

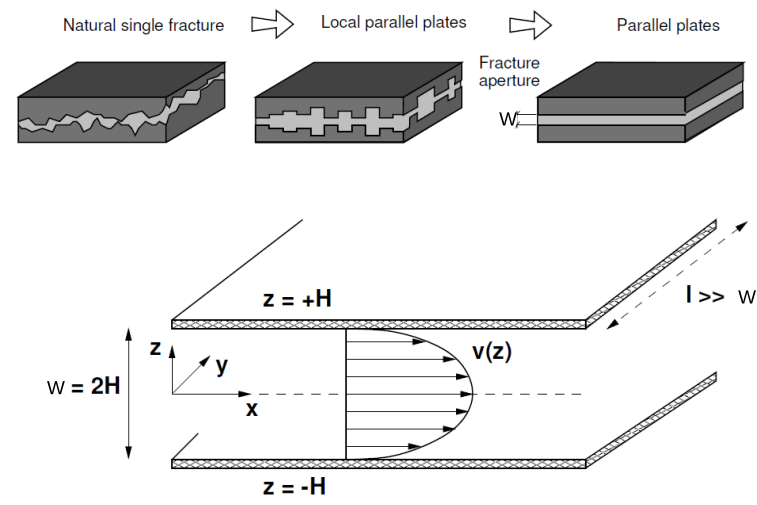

Figure 2: Parallel-Plate Concept

$$
Q=-\left(\frac{l w^{3}}{12 \mu}\right) \nabla P
$$

The similarity of the cubic law with the Darcy equation (equation 9) leads to the following definition of the intrinsic permeability $k=\frac{w^{2}}{12}$.

If we consider a fractured element with a crack aperture $w$ in the $\mathrm{Z}$ direction. The hydraulic behavior should be described by an anisotropic permeability tensor. The volumetric flow in the $\mathrm{Z}$ directions (Figure 2 ) is not modified and is defined by the Darcy law with a permeability coefficient of the uncracked material. If we consider $e_{x}$ and $e_{y}$ the two orthonormal vectors in the crack plane, and $e_{z}$ the outward unit normal to this plane the fluid-flow governing equation reads

$$
Q . e_{z}=-k \frac{A_{z}}{\mu} \nabla P . e_{z}
$$

where $A_{z}$ is the cross sectional area in the $\mathrm{Z}$ direction.

For the $\mathrm{X}$ and $\mathrm{Y}$ directions, in the uncracked solid matrix, the flow is also described by the Darcy law

$$
\begin{aligned}
& Q . e_{x}=-k \frac{A_{x}}{\mu} \nabla P . e_{x} \\
& Q . e_{y}=-k \frac{A_{y}}{\mu} \nabla P . e_{y}
\end{aligned}
$$

$A_{x}$ and $A_{x}$ are the cross areas in the directions $X$ and $Y$.

The quantities flowing through the crack are given by

$$
\begin{aligned}
& Q . e_{x}=-\left(\frac{L_{y} w^{3}}{12 \mu}\right) \nabla P . e_{x} \\
& Q . e_{y}=-\left(\frac{L_{x} w^{3}}{12 \mu}\right) \nabla P \cdot e_{y}
\end{aligned}
$$

These equations are similar to the Darcy ones with a cracked cross area $A_{c r}^{x}=L_{y} w$ and $A_{c r}^{y}=L_{x} w$. Equation 19 reads

$$
\begin{aligned}
& Q . e_{x}=-\frac{w^{2}}{12} \frac{A_{c r}^{x}}{\mu} \nabla P . e_{x} \\
& Q . e_{y}=-\frac{w^{2}}{12} \frac{A_{c r}^{y}}{\mu} \nabla P . e_{y}
\end{aligned}
$$

The intrinsic permeability coefficient is $w^{2} / 12$. So we have two kind of transport-law equations driving the flowing through the cracked volume. The first ones govern the flow through the uncracked matrix and is given by

$$
\begin{aligned}
& Q_{0} \cdot e_{x}=-k \frac{L_{y} L_{z}}{\mu} \nabla P . e_{x}=-k \frac{A_{x}}{\mu} \nabla P . e_{x} \\
& Q_{0} \cdot e_{y}=-k \frac{L_{x} L_{z}}{\mu} \nabla P . e_{y}=-k \frac{A_{y}}{\mu} \nabla P . e_{y} \\
& Q_{0} \cdot e_{z}=-k \frac{L_{x} L_{y}}{\mu} \nabla P . e_{z}=-k \frac{A_{z}}{\mu} \nabla P . e_{z}
\end{aligned}
$$


and those driving the flowing through the fracture

$$
\begin{aligned}
& Q_{F} \cdot e_{x}=-\left(\frac{L_{y} w^{3}}{12 \mu}\right) \nabla P \cdot e_{x} \\
& Q_{F} \cdot e_{y}=-\left(\frac{L_{x} w^{3}}{12 \mu}\right) \nabla P \cdot e_{y} \\
& Q_{F} \cdot e_{z}=0
\end{aligned}
$$

We could express the last equations in terms of the total cross area $A$ in each direction $\left(A_{x}=\right.$

$$
\begin{aligned}
L_{y} L_{z}, A_{y} & \left.=L_{x} L_{z}, A_{z}=L_{x} L_{y}\right) \\
Q_{F} \cdot e_{x} & =-\left(\frac{L_{y} w^{3}}{12 \mu}\right) \nabla P \cdot e_{x}=-\frac{w^{3}}{12 L_{z}} \frac{A_{x}}{\mu} \nabla P \cdot e_{x} \\
Q_{F} \cdot e_{y} & =-\left(\frac{L_{x} w^{3}}{12 \mu}\right) \nabla P \cdot e_{y}=-\frac{w^{3}}{12 L_{z}} \frac{A_{y}}{\mu} \nabla P \cdot e_{x} \\
Q_{F} \cdot e_{z} & =0 .
\end{aligned}
$$

Therefore, in the orthotropic plan of the crack, the fractured permeability tensor is given by

$$
\left[\begin{array}{ccc}
k_{0}+\frac{w^{3}}{12 L_{z}} & 0 & 0 \\
0 & k_{0}+\frac{w^{3}}{12 L_{z}} & 0 \\
0 & 0 & k_{0}
\end{array}\right]
$$

$k_{0}$ is the initial permeability of the the uncracked material.

The preceding permeability tensor has been developed considering one fracture in the $Z$ direction. If we consider a general case where the concrete element develops cracks in the three directions, the following tensor should be considered to be added to the initial permeability tensor.

$$
\left[\begin{array}{ccc}
\frac{w^{3}}{12 L_{z}}+\frac{w^{3}}{12 L_{y}} & 0 & 0 \\
0 & \frac{w^{3}}{12 L_{z}}+\frac{w^{3}}{12 L_{x}} & 0 \\
0 & 0 & \frac{w^{3}}{12 L_{y}}+\frac{w^{3}}{12 L_{x}}
\end{array}\right]
$$

\section{Validation}

\subsection{D Numerical macroscopic modelling of the splitting test}

In [15], an experimental procedure has been performed in order to relate in real-time the water permeability and the crack openingsdisplacements in a saturated concrete speci-

\begin{tabular}{|c|c|c|c|c|}
\hline$f_{t}(M P a)$ & \multicolumn{2}{|c|}{$E(G P a)$} & $G_{f}(N / m)$ & $k\left(m^{2}\right)$ \\
\hline 3 & & 47 & 80 & $10^{-21}$ \\
\hline $\bar{\mu} \mu(P a . s)$ & $\bar{B}$ & $M(G$ & & \\
\hline $10^{-3}$ & 0.3 & 10 & & \\
\hline
\end{tabular}
mens. The permeability test is performed simultaneously with the mechanical loading. Figure 3 shows the configuration of the test setup. More details are given in [15] [16].

The numerical simulation are performed using the poromecanical-damage based model proposed. Table 1 gives the values of the model parameters used in simulations

Table 1: Numerical Model Parameters for the Splitting test simulation

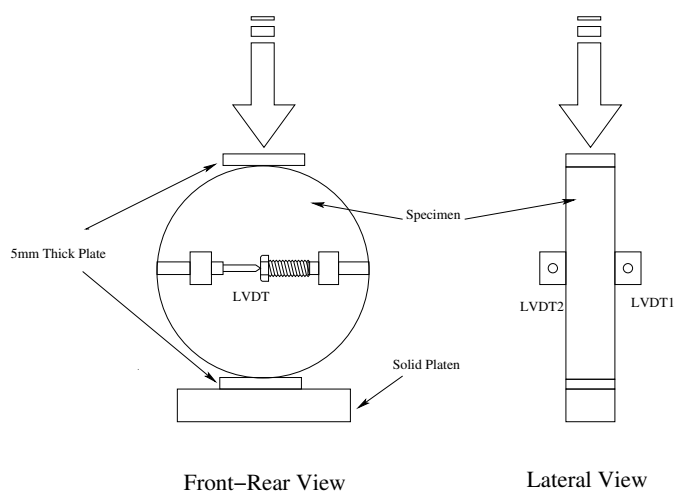

Figure 3: Splitting Test Setup

The computation is performed in $3 \mathrm{D}$, and the boundary condition are applied in order to be as close as possible of the experimental conditions. The field of crack openings computed at the end of the loading are presented at figure 4.

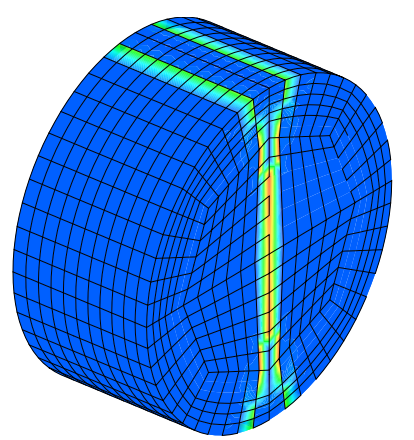

Figure 4: Field of crack openings 


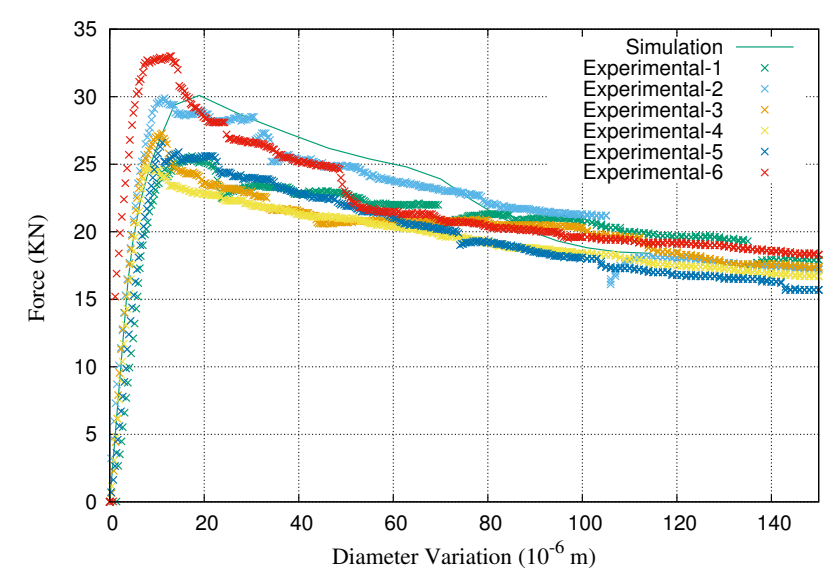

Figure 5: Load-Displacements response of the hydromechanical test : Experimental Vs Numerical results

The global behavior represented in Figure 5 gives the evolution of the Load Vs the Diameter variation of the cylinder. The numerical simulation result is compared to the experimental ones. Experimentally, six specimens were tested. The numerical results approximates well both the peak load and the post-peak behavior.

Regarding the evolution of the "apparent" permeability, experimentally, this permeability is estimated according to the Darcy's law under the assumption that the percolation crosssection area is that one with the diameter $77 \mathrm{~mm}$ (The sample effective surface). Numerically, the finite element computation gives numerical values of the intrinsic permeability at integrations points. In order to estimate the "apparent" permeability using the Darcy's law, the volumetric flux resulting from the finite element computation is computed and reduced on the effective area $(d=77 \mathrm{~mm})$. Before discussing the numerical results about the apparent permeability, it is very important to mention that in the cubic law based on the Parallel-Plate Concept a constant crack aperture is assumed for the total fracture surface (Figure 2). The latter approach is a very strong hypothesis. The crack roughness, aperture variation and tortuosity effect are not taken into account. Many authors [19] [8] introduces an empirical coefficient $\xi$ to correct the standards cubic law

$$
Q=-\xi\left(\frac{l w^{3}}{12 \mu}\right) \nabla P
$$

Some authors proposed a constant value of $\xi$. In [15], this empirical coefficient is no longer considered as a constant parameter, but it depends on the crack aperture and the following form is proposed $\xi=\min \left(\alpha w^{\gamma}, 1\right)$ with $\alpha=5.62510^{-5} \mathrm{~m}$ and $\gamma=1.19$. For the sake of unit homogeneity, we propose the equivalent formulation which is not unit dependent :

$$
\xi=\left(\frac{w}{w_{f}}\right)^{\gamma} i f w<w_{f}
$$

with $w_{f}=228.3 \mu \mathrm{m}$ Figure 6 shows the evolution of the normalized apparent permeability $k / k_{0}$ for both experiments and computations. The good agreement shows that the model is correctly implemented into the Finite Element code.

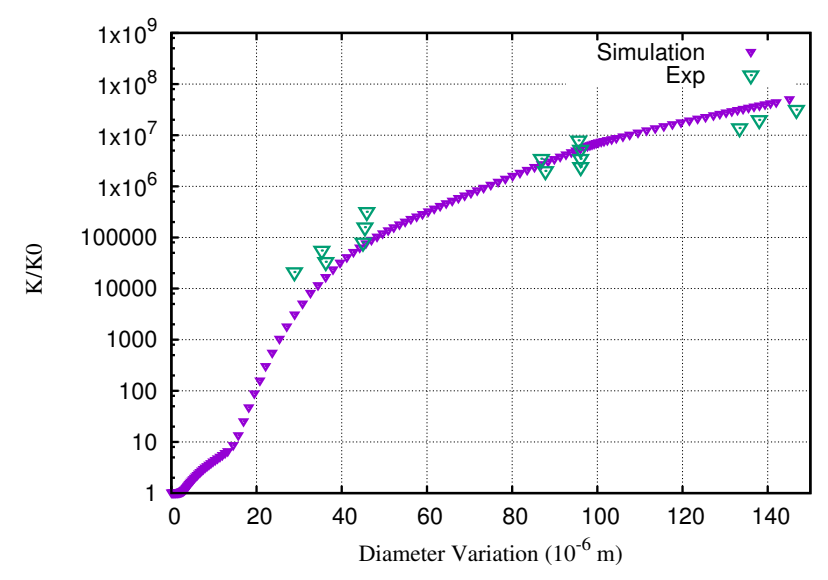

Figure 6: Evolution of the normalized permeability : Experimental Vs Numerical results

\subsection{Mesoscopic modelling}

The previous approach is now used at the mesoscopic scale. The concrete is no more considered as homogeneous but as a structure discretized at the scale of sand grain. The method used, described in previous papers [2, 14], allows to better describe the cracking process zone which may influe on the permeability in particular when the cracks are not completely localized. In order to keep computation time reasonable, a $2 \mathrm{D}$ approach is plane stresses in used. The mesh, the damage field an crack opening at the end of computation are pictured at figure 7 

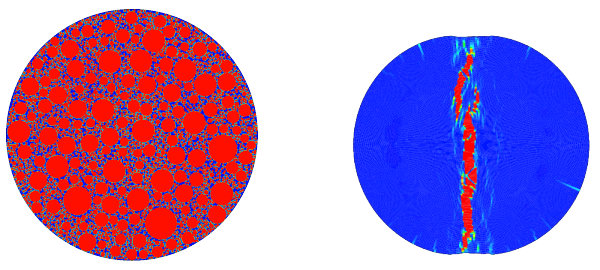

Figure 7: mesoscopic mesh, crack width

The evolution of relative permeability are reported at figure 8 for two hypothesis :

- Pure Poiseuille law (equ. 16)is used for the cracks (WO $\xi$ )

- The flow coefficient $\xi$ defined at equ. 27 is used

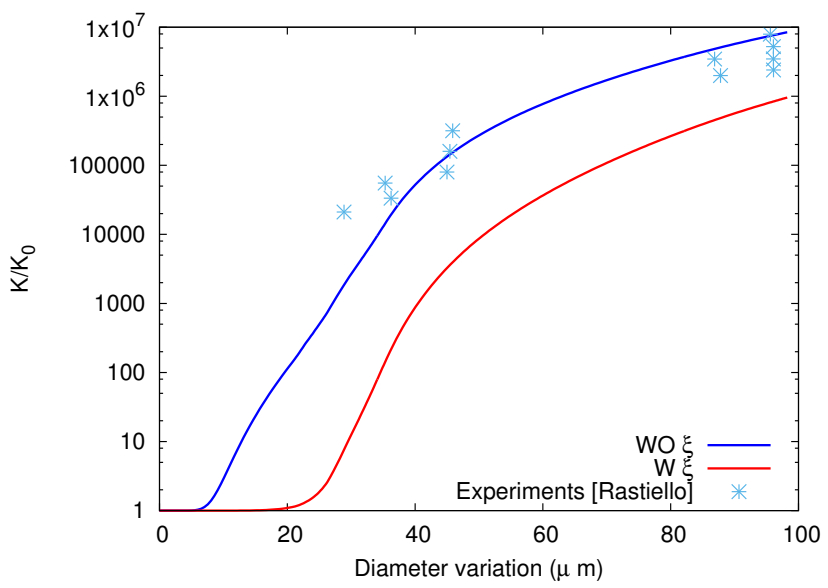

Figure 8: Evolution of the normalized permeability, mesoscopic computation

The results shows that the computation is in good agreement with the experiments when using directly the Poiseuille law. The use of the flow coefficient at the mesoscopic level drives to underestimate the permeability of on order of magnitude.

\section{CONCLUSIONS}

In the present paper, a damageporomechanical approach has been proposed to carry out the crack-permeability interaction in fractured concrete. A 3D macroscopic modelling of a splitting test where the permeability test is performed simultaneously with the application of the mechanical load was considered.
The first validations proved the capability of the proposed model to reproduce the hydromechanical behavior interdependent. Future works will be related to the investigation of the tortuosity effect and the interaction of the Biot coefficient with the cracking process.

\section{REFERENCES}

[1] M. Choinska, A. Khelidj, G. Chatzigeorgiou, and G. Pijaudier-Cabot. Effects and interactions of temperature and stresslevel related damage on permeability of concrete. Cement and Concrete Res., 37:79-88, 2007.

[2] Atef Daoud, Olivier Maurel, and Christian La Borderie. 2d mesoscopic modelling of barconcrete bond. Engineering Structures, 49:696-706, April 2013.

[3] H. Darcy. Recherches experimentales relatives au mouvement de l'eau dans les tuyaux. Mallet-Bachelier, 1857.

[4] L. Dormieux and D. Kondo. Approche micromécanique du couplage perméabilitéendommagement.

C.R. Mecanique, 332:135-140 (in French), 2004.

[5] F. Dufour, G. Pijaudier-Cabot, M. Choinska, and A. Huerta. Permeability due to the increase of damage in concrete : from diffuse to localised damage distributions. $J$ ENG MECH-ASCE, 135(9):1022-1028, 2009.

[6] S. Fichant, C. La Borderie, and G. Pijaudier-Cabot. Isotropic and anisotropic description of damage in concrete structures. Mech Cohes Frict Mater, 4:339-359, 1999.

[7] B. Gérard, D. Breysse, A. Ammouche, O. Houdusse, and O. Didry. Cracking and permeability of concrete under tension. Mater. and Struc., 29(3):141-151, 1996. 
[8] S. Grasberger and G. Meschke. Thermohygro-mechanical degradation of concrete: From coupled 3d material modelling to durability-oriented multifield structural analyses. Mater. Struct., 37:244-256, 2004.

[9] M. Matallah, M. Farah, F. Grondin, A. Loukili, and E. Rozire. Sizeindependent fracture energy of concrete at very early ages by inverse analysis. Eng. Fract. Mech., 109:1-16, 2013.

[10] M. Matallah and C. La Borderie. Inelasticity-damage-based model to numerical modelling of concrete cracking. Engng Frac Mech, 76:1087-1108, 2009.

[11] M. Matallah, C La Borderie, and O. Maurel. A practical method to estimate crack openings in concrete structures. Int $J$ Nume Anal Met, 34:1615-1633, 2010.

[12] H. Meziani and F. Skoczylas. An experimental study of the mechanical behaviour of a mortar and of its permeability under deviatoric loading. Mater. and Struc., 32:403-409, 1999.

[13] A. Michou, A. Hilaire, F. Benboudjema, G. Nahas, P. Wyniecki, and Y. Berthaud. Reinforcement concrete bond behavior: Experimentation in drying conditions and meso-scale modeling. Eng Struct, 101:570-582, 2015.

[14] D. Nguyen, C. Lawrence, C. La Borderie, M. Matallah, and G. Nahas. A mesoscopic model for a better understanding of the transition from diffuse damage to localized damage. EJECE, 14:751-776, 2010.
[15] G. Rastiello, C. Boulay, J. L. Tailhan, and P. Rossi. Real-time water permeability evolution of a localized crack in concrete under loading. Cement Concrete Res, 56:20-28, 2014.

[16] G. Rastiello, J. L. Tailhan, P. Rossi, and S. Dal Pont. Macroscopic probabilistic cracking approach for the numerical modelling of fluid leakage in concrete. Ann. Solid Struct., DOI 10.1007/s12356-0150038-6, 2015.

[17] E. Romm. Flow characteristics of fractured concrete (in Russian). Nedra, Moscow, 1966.

[18] J. Saliba, F. Grondin, M. Matallah, A. Loukili, and H. Boussa. Relevance of a mesoscopic modeling for the coupling between creep and damage in concrete. Mechanics of Time-Dependent Materials, 17:481-499, 2012.

[19] S. Secchi and B. Schefler. A method for 3-d hydraulic fracturing simulation. Int. J. Fract., 178(1):245-258, 2012.

[20] D. Snow. Anisotropic permeability of fractured media. Water Resources Research, 5(6):1273-1289, 1969.

[21] K. Wang, D.C. Jansen, S.P. Shah, and A.F. Karr. Permeability study of cracked concrete. Cement and Concrete Research, 2(3):141-151, 1997.

[22] F. M. White. Fluid Mechanics. McGrawHill, New York, 4th ed. edition, 1999. 